\title{
156
}

\section{A Boundary-Layer Problem Associated with Magnetogasdynamic Channel Flow}

\author{
F. E. C. CuLICK* \\ California Institute of Technology, Pasadena, Calif.
}

$\mathbf{I}^{\mathbf{N}}$ $\mathrm{N}$ connection with the motion of electrically conducting fluids through electric and magnetic fields, it is useful to consider flow in a channel as a possible means of energy extraction or addition. The simplest form of analysis, based on the familiar one-dimensional approximation, excludes the very important influences of viscous stresses and heat flux. Although one may account for surface friction and heat transfer in an approximate manner as Dahlberg ${ }^{1}$ has discussed, there is no possibility for computing the detailed effects. One way of (partially) correcting this defect is based on the idea that, for some distance downstream of the entrance, the immediate effects of the walls may be confined to thin boundary regions. The central portion of the flow is regarded as a one-dimensional problem, the solution of which provides the "freestream" conditions set in the boundary-layer problem.

Although there have been a number of discussions of boundary layers on plates when the fluid is electrically conducting, there seems to have been much less work on problems arising in channel flow. The closest to the present discussion is that by Kerrebrock ${ }^{2}$ and Hale, ${ }^{3}$ who treat special cases; the latter incorporates Hall currents, which are ignored here. Moffat ${ }^{4}$ has discussed the problem and used an integral method to analyze the boundary layers on the side (insulating) walls but with the pressure constant.

The results outlined here are restricted to the necessary conditions for the existence of a class of similarity solutions to a particular set of boundary-layer equations for compressible flow. The formulation is based on certain assumptions that have been discussed in detail, for example, in Refs. 2 and 5 . It is necessary only to state the conventions adopted; the electric field $E$, magnetic induction $B$, and "freestream" flow speed $u$ are positive in the negative $z$, positive $y$, and positive $x$ directions, respectively. The current density $j$ is then positive in the positive $z$ direction for a generator. It is supposed that $E$ and $B$ are established by means external to the flow so that the local current density in a generator is, for a scalar electrical conductivity,

$$
j=\sigma(u B-E)
$$

If the "magnetic Reynolds number" is small, then the magnetic field associated with the flow of currents in the gas is

Received February 11, 1963; revision received July 17, 1963. This work was supported by the Air Force Office of Scientific Research under Contract AF 49(638)758 with California Institute of Technology.

* Assistant Professor of Jet Propulsion. small, and it is consistent to assume $B$ to be caused ess $\mathrm{n}$. tially by external means only. The corresponding assur.ıption that the electric field strength $E$ also is due to sources outside the flow implies that the gas should be electrically neutral at all points. This situation often prevails because of the large forces that arise if there is significant charge separation. However, in the present problem, somewhat closer examination is necessary. Consider the boundary layers on the electrodes of a generator; away from the regions near the side (insulating) walls, the current must be uniform in the direction normal to the surface since no current flow is permitted, within the approximations adopted here, in the axial directions. Thus, since $u$ and $\sigma$ vary through the boundary layer, Eq. (1) can be satisfied only if $E$ varies as well. This means that there is space charge within the boundary layer, and the drop in potential across the boundary layer is different from that in an equal distance in the freestream. Equation (1), applied within the boundary layer, is really an equation determining $E$. The contribution of net charge density, $\epsilon_{0} \nabla \cdot \mathrm{E}$, to the current flow is a small correction that may be neglected.

On the other hand, the electric field can be uniform within the boundary layers on the side walls except in the regions near the electrodes. One can suppose that the tendency to charge neutrality does prevail and that Eq. (1) is an equation for $j$ in the viscous region. Clearly, at all points for which $u B<E$ ( $u \rightarrow 0$ near the surface, of course), $j$ is locally negative, and current flows in the direction opposite to that of the current outside the boundary layer. Hence, there exists the possibility for closed-current loops within the channel. The flow in the corners, where the boundary layers on the electrodes and the side walls merge, constitutes a very much more difficult problem that has not been investigated further. It may be a practically important question because of the concomitant power losses.

The situation in an accelerator is different, for $u B<E$ everywhere; the current flows in the same direction at all points. However, since $|j|=\sigma|E-u B|$ and both $u$ and $1 / \sigma$ decrease in the region near the side walls, $|j|$ must increase. The flow adjacent to these walls, therefore, offers a "short circuit" path relative to the flow in the central region of the channel. These remarks indicate that the solutions to the boundary-layer equations for the flow over the side walls and electrodes should differ in a qualitative, as well as quantitative, respect, and indeed, one such distinction appears already in the similarity solutions enumerated below.

It is assumed that the fluid behaves as a perfect gas with constant Prandtl number and specific heats. The equations of conservation for the flow outside the boundary layers are those appropriate to a one-dimensional flow; the corresponding boundary-layer equations can be deduced from the Navier-Stokes equations written for an electrically conducting fluid and may be found, for example, in Ref. 3. Following the usual approach, one seeks conditions under which the boundary-layer partial differential equations may be reduced to nonlinear, ordinary differential equations; all 
Table la Summary of channel flow solutions for boundary-layer similar flows on electrodes ( $E_{e}$ constant)

\begin{tabular}{cll}
\hline \hline & Energy equation & Momentum equation \\
\hline $\begin{array}{c}\text { Constant } \\
\text { enthalpy }\end{array}$ & $n=-E_{e} j_{r} \frac{A_{r} x_{r}}{u_{r}^{2} \rho_{e} u_{e} A}$ & $\frac{u_{e}}{u_{3}}=1-\left[1+\frac{\alpha(1-3 n)}{(5 n-1)}\right] \frac{(5 n-1)}{n(1+\alpha)} \frac{1}{\gamma M_{e}^{2}}$ \\
$\begin{array}{c}\text { Constant } \\
\text { velocity }\end{array}$ & $c=-E_{e} j_{r}(1+\alpha) \frac{A_{r} x_{r}}{u_{r}^{2} \rho_{e} u_{e} A}$ & $\frac{u_{e}}{u_{3}}=\left(\frac{1-\alpha}{c}\right) \frac{\gamma-1}{\gamma}$ \\
$\begin{array}{c}\text { Constant } \\
\text { Mach no. }\end{array}$ & $\lambda=-E_{e} j_{r} \frac{A_{r} x_{r}(1+\alpha)}{2 h_{r} \rho_{e} u_{e} A\left\{1+[(\gamma-1) / 2] M_{e}^{2}\right\}}$ & $\frac{u_{e}}{u_{3}}=\frac{M_{e}^{2}+[(1-\lambda-\alpha) / \lambda \gamma]}{M_{e}{ }^{2}+[2 /(\gamma-1)]}$ \\
\hline \hline
\end{tabular}

Table 1b Exponents $\beta$ in the distribution $\psi=\psi_{r}\left(x / x_{r}\right)^{\beta}$

\begin{tabular}{lccc}
\hline \hline & $\begin{array}{c}\text { Constant } \\
\text { enthalpy }\end{array}$ & $\begin{array}{c}\text { Constant } \\
\text { velocity }\end{array}$ & $\begin{array}{c}\text { Constant } \\
\text { Mach no. }\end{array}$ \\
\hline$u_{e}$ & $n$ & $\frac{c}{1+\alpha}$ & $\frac{\lambda}{1+\alpha}$ \\
$M_{e}$ & $n$ & $\frac{c}{2(1+\alpha)}$ & $\frac{2 \lambda}{1+\alpha}$ \\
$p_{e}$ & $\frac{1-5 n-\alpha(1+n)}{1+\alpha}$ & $\frac{1-\alpha}{1+\alpha}$ & $\frac{1-\lambda-\alpha}{1+\alpha}$ \\
$\rho_{e}$ & $\frac{1-5 n-\alpha(1+n)}{1+\alpha}$ & $\frac{1-\alpha-c}{1+\alpha}$ & $\frac{1-3 \lambda-\alpha}{1+\alpha}$ \\
$A$ & $\frac{4 n-1-\alpha(1+2 n)}{1+\alpha}$ & $\frac{c-1+\alpha}{1+\alpha}$ & $\frac{2 \lambda-1+\alpha}{1+\alpha}$ \\
$j$ & $\frac{-2[n+\alpha(1-n)]}{1+\alpha}$ & $\frac{-2 \alpha}{1+\alpha}$ & $\frac{-2 \alpha}{1+\alpha}$ \\
& $\frac{-2 \alpha(1-2 n)}{1+\alpha}$ & $\frac{-2 \alpha}{1+\alpha}$ & $\frac{-2 \alpha}{1+\alpha}$ \\
\hline$\sigma_{e}$ & $\frac{1+2 \alpha}{1+\alpha}$ &
\end{tabular}

dependent variables are then functions of a single variable, and the problem of solution is posed in simplest form. The transformation used here is the familiar stretching of the coordinate normal to the surface, $z \rightarrow \eta$, with $d \eta=$ $\left(\rho / \rho_{\odot}\right) t(x) d z, t(x)$ being a function of $x$ to be determined. Then the class of boundary-layer solutions found are those for which the stream function has the separated form, $\zeta(x) f(\eta)$, with $\zeta(x)$ another function of $x$ to be determined. Simultaneously, the properties of the inviscid flow must also be taken as functions of $x$ consistent with the one-dimensional flow equations. The various functions of $x$ are determined by the requirements of similarity and satisfaction of the equations for the inviscid flow.

One finds eventually that, for the boundary layers on the electrodes, there are three subclasses of the similarity solutions corresponding to constant enthalpy, velocity, and Mach number. The associated channel flow solutions are summarized in Tables $1 \mathrm{a}$ and $1 \mathrm{~b}$, in which subscript $e$ denotes quantities in the one-dimensional flow and subscript $r$ denotes reference values. For the case quoted, the electric field has been assumed uniform, and the coefficient of viscosity is proportional to the absolute temperature; these restrictions may be relaxed so long as $E_{\text {c }}$ and $\mu_{*}$ vary as powers of $x . \dagger$ One does not have complete freedom in choosing the constants $\lambda, c, n$, and $\alpha$, essentially because of the requirement that $u_{e} / u_{3}$ $>1$ in a generator and $u_{a} / u_{3}<1$ is an accelerator; ${ }^{6} u_{3}=E$ / $B$ is generally a function of $x$. A summary of the permissible ranges appears in Table 2.

Calculations appropriate to the boundary layers on the side walls can be carried out in a similar manner, but account must be taken that the current density is variable through the boundary layer. It turns out that this further requirement is satisfied only by the similarity solutions for which the "freestream" Mach number is constant. Numerical solutions for this case may, therefore, be the most interesting of those in Table 1.

One might consider the possibility of seeking similar solutions for very large values of $M_{e}$ by using the stagnation enthalpy rather than the static enthalpy as the dependent variable in the energy equation. It is quite easy to show, however, that there are no such solutions for $M_{c} \rightarrow \infty$. The difficulty encountered by Kerrebrock ${ }^{2}$ arises in all three classes of solutions. No attempt has been made to treat segmented electrodes, which is consistent with the neglect of Hall currents. Similarity solutions cannot be obtained if

Table 2 Summary of permissible ranges of $\alpha, n, c, \lambda$, for boundary-layer flows on electrodes ( $E_{e}$ constant)

\begin{tabular}{|c|c|c|c|c|}
\hline \multirow[b]{2}{*}{ Constant enthalpy } & \multicolumn{2}{|c|}{ Generator $\left(u_{e} / u_{3}>1\right)$} & \multicolumn{2}{|c|}{ Accelerator $\left(u_{0} / u_{3}<1\right)$} \\
\hline & $\alpha<-1:$ & $n<0$ & $\alpha<-1:$ & $0<n<\frac{1-\alpha}{5-3 \alpha}$ \\
\hline & $1<\alpha<\frac{5}{3}$ & $\frac{\alpha-1}{3 \alpha-5}<n<0$ & $-1<\alpha \leqslant+1:$ & $n>\frac{1-\alpha}{5-3 \alpha}$ \\
\hline$\alpha \neq-1$ & $\alpha>\frac{5}{3}:$ & $n<0$ & $\begin{array}{c}1<\alpha \leqslant \frac{5}{3} \\
\alpha \geqslant \frac{5}{3}\end{array}$ & $\begin{array}{c}n>0 \\
n<\frac{\alpha-1}{3 \alpha-5}\end{array}$ \\
\hline Constant velocity & $\alpha<-1:$ & $0<c<\frac{\gamma-1}{\gamma}(1-\alpha)$ & & \\
\hline $\begin{array}{l}\lambda=0 \\
\alpha \neq 1\end{array}$ & $\alpha>+1:$ & $-\frac{\gamma-1}{\gamma}(\alpha-1)<c<0$ & $-1<\alpha<+1$ : & $c>\frac{\gamma-1}{\gamma}(1-\alpha)$ \\
\hline \multirow[t]{2}{*}{ Constant Mach number } & $\alpha<-1:$ & $0<\lambda<\frac{\gamma-1}{3 \gamma-1}(1-\alpha)$ & $\alpha<-1:$ & $\lambda<0$ \\
\hline & & & $-1<\alpha+1:$ & $\lambda>\frac{\gamma-1}{3 \gamma-1}(1-\alpha)$ \\
\hline$\alpha \neq-1$ & $\alpha>+1$ & $\lambda<-\frac{\gamma-1}{3 \gamma-1}(\alpha-1)$ & $\alpha \geqslant 1:$ & $\lambda>0$ \\
\hline
\end{tabular}


$E$ and $j$ vary discontinuously, but it might be possible to use numerical solutions as a basis for an integral method, as difficult boundary conditions have been handled in other problems.

\section{References}

${ }^{1}$ Dahlberg, E., "On the one-dimensional flow of a conducting gas in crossed fields," Quart. Appl. Math. XIX, 177-193 (1961).

2 Kerrebrock, J. L., "Electrode boundary layers in direct current plasma accelerators," J. Aerospace Sci. 28, 631-643 (1961).

${ }^{3}$ Hale, F. J., "Insulator boundary layers in magnetohydrodynamic channels," Mass. Inst. Tech., Ph.D. Thesis, Dept. Aeronaut. and Astronaut. (1962).

4 Moffatt, W. C., "Boundary layer effects in magnetohydrodynamic flows," Magnetogasdynamic Lab., Dept. Mech. Eng., Mass. Inst. Tech. Rept. 61-4 (1961).

' Resler, E. L., Jr. and Sears, W. R., "The prospects for magneto-aero-dynamics," J. Aeronaut. Sci. 25, 235-248 (1958).

${ }^{6}$ Culick, F. E. C., "Magnetogasdynamic channel flow and convective heat transfer," Calif. Inst. Tech., Daniel and Florence Guggenheim Jet Propulsion Center, TN 6 (1962). 